\title{
WREATH PRODUCTS AND EXISTENTIALLY COMPLETE SOLVABLE GROUPS
}

\author{
BY \\ D. SARACINO( $\left.{ }^{1}\right)$
}

\begin{abstract}
It is known that the theory of abelian groups has a model companion but that the theory of groups does not. We show that for any fixed $n \geq 2$ the theory of groups solvable of length $\leq n$ has no model companion. For the metabelian case $(n=2)$ we prove the stronger result that the classes of finitely generic, infinitely generic, and existentially complete metabelian groups are all distinct. We also give some algebraic results on existentially complete metabelian groups.
\end{abstract}

0. Introduction. In 1970, Eklof and Sabbagh [2] showed that the theory $T_{1}$ of abelian groups has a model companion, but that the theory $T$ of groups does not. Shortly afterward, A. Macintyre [5] strengthened the negative result for groups by showing that the class of existentially complete groups is distinct from the class of infinitely generic ones. In this paper we consider analogous questions for some theories of groups intermediate between the extremes represented by $T_{1}$ and $T$.

Specifically, for each integer $n \geq 1$, let $T_{n}$ be the theory of groups solvable of length $\leq n$. (Some of the model-theoretic and group-theoretic terminology relevant to this paper will be recalled in $\$ 1$.) Thus $T_{1}$ gives abelian groups, $T_{2}$ gives metabelian groups, and so on. Our main results are the following:

Theorem 1. For any $n \geq 2, T_{n}$ has no model companion.

For the case $n=2$, we have a stronger result:

Theorem 2. There is an $\exists \forall \exists$ sentence of first-order group theory which holds in every infinitely generic metabelian group and fails in every finitely generic metabelian group. Thus no metabelian group is both finitely generic and infinitely generic, so in particular the class of existentially complete metabelian groups is distinct from the class of infinitely generic ones and from the class of finitely generic ones.

Presented to the Society, March 26, 1973 under the title Existentially complete solv. able groups; received by the editors July 13, 1973.

AMS (MOS) subject classifications (1970). Primary 02H05, 02H15; Secondary 20E15, $20 \mathrm{~F} 25$.

Key words and phrases. Model companion, wreath product, ultraproduct, finite forcing, infinite forcing, existential completeness.

( $\left.{ }^{1}\right)$ Research supported in part by National Science Foundation Grant GP-34088.

Copyright $\odot$ 1974, American Mathematical Society 
We also give some further results on existentially complete metabelian groups. For example, we show that if $G$ is any finitely generic metabelian group and $G^{(1)}$ its commutator subgroup, then $G / G^{(1)}$ is periodic, i.e. contains no elements of infinite order. We also show that there exists a finitely generic metabelian group which is itself periodic. By way of contrast, if $G$ is an infinitely generic metabelian group, then $G / G^{(1)}$ is never periodic.

1. Preliminaries. We assume that the reader is familiar with the basic notions of first-order model theory, and with the notions of finite and infinite forcing in model theory, finitely and infinitely generic structures, and finite and infinite forcing companions. (The fundamental papers are [7] and [8].) We recall that if $L$ is a first-order language and $\Sigma$ a class of $L$-structures then a structure $A \in \Sigma$ is said to be existentially complete in $\Sigma$ if for any $B \in \Sigma$ which extends $A$ and any $L$-formula $\psi$ of the form $\exists x_{1} \cdots \exists x_{n} \phi$, where $\phi$ is quantifier-free and has constants from $A, B \vDash \psi$ implies $A \vDash \psi$. If $K$ is a theory in $L$, then a theory $K^{\prime}$ is said to be a model companion of $K$ if $K^{\prime}$ is model-complete (see [9]) and $K$ and $K^{\prime}$ are mutually model-consistent, i.e. any model of $K$ can be embedded in a model of $K^{\prime}$ and vice-versa. (Model companions are introduced in [2]. If $K_{1}^{\prime}$ and $K_{2}^{\prime}$ are model companions of $K$, then $K_{1}^{\prime}$ and $K_{2}^{\prime}$ have the same models; this is proved in [1], but the essential idea is already in [9].) If $\Sigma$ is the class of all substructures of models of $K$, i.e. the class $\operatorname{Mod}\left(K_{\forall}\right)$ of models of the set of universal consequences of $K$, then $K$ has a model companion iff the class of existentially complete structures in $\Sigma$ is elementary in the wider sense (see [2]); thus in particular to establish that a theory $K$ has no model companion it is sufficient to show that the class of existentially complete structures in $\operatorname{Mod}\left(K_{\forall}\right)$ is not closed under ultrapowers.

For definiteness, we axiomatize the theory $T$ of groups in a first-order language with equality which has a binary function symbol $o$, a unary function symbol -1 , and a constant symbol 1 (intended to denote respectively group multiplication, inverses, and the identity element). It is easy to write down a set $T$ of universal sentences in this language which axiomatizes the theory of groups.

In order to describe the theories $T_{n}$, we recall that if $a$ and $b$ are elements of a group $G$ then the ir commutator $[a, b]$ is defined as the element $a^{-1} b^{-1} a b$ of G. The derived (or commutator) subgroup $G^{(1)}$ of $G$ is the subgroup of $G$ generated by $\{[a, b]: a, b \in G\}$. The derived subgroup of $G^{(1)}$ is then denoted by $G^{(2)}$; the derived subgroup of $G^{(2)}$ is denoted by $G^{(3)}$, and so on. Each $G^{(n)}$ is a normal subgroup of $G, G^{(n)} / G^{(n+1)}$ is abelian for each $n$, and we have $G G^{(1)}$ ' $G^{(2)} \supset \ldots$. If $G^{(n)}=\{1\}$, the trivial subgroup, $G$ is said to be solvable of length $\leq n$. If we agree to define a second commutator as the commutator of two commutators, a third commutator as the commutator of two second commutators, and so on, 
then it is easy to see that $G$ is solvable of length $\leq n$ iff every $n$th commutator is 1. It is clear that this latter condition is expressible by a universal sentence $\phi_{n}$ of our language. If we define $T_{n}$ as $T \cup\left\{\phi_{n}\right\}$, then each $T_{n}$ is a set of universal sentences, and the models of $T_{n}$ are precisely the groups solvable of length $\leq n$.

The principal algebraic tool of this paper is the standard unrestricted wreath product (see, e.g., [10]). Let $A$ and $B$ be groups. Recall that the Cartesian power $A^{B}$ is the set of functions from $B$ to $A$, with multiplication defined componentwise: $f g(b)=f(b) g(b)$ for all $b \in B$. If $B$ acts on itself by right multiplication then we have an induced action on the Cartesian power; if $f \in A^{B}$ and $b \in B$, then the result $f^{b}$ of $b$ 's acting on $f$ is given by $f^{b}(a)=f\left(a b^{-1}\right)$ for $a \in B$. The semidirect product of $A^{B}$ and $B$, with this action, is denoted by $A \operatorname{Wr} B$ and called the standard unrestricted wreath product of $A$ and $B$. The elements of $A W_{\mathbf{r}} B$ are pairs $(b, f)$ with $b \in B$ and $f \in A^{B}$; and we have $(b, f)(c, g)=$ $\left(b c, f^{c} g\right)$. When it is convenient we identify $A^{B}$ with the set of elements of the form $(1, f)$, and $B$ with the set of elements of the form $(b, 1)$; thus we sometimes write the element $(b, f)$ as $b f$. We observe that if $A$ and $B$ are abelian, then $A W_{\mathrm{r}} B$ is metabelian; more generally, if $A$ and $B$ are solvable of lengths $\leq m$ and $\leq n$, respectively, then $A W_{\mathrm{r}} B$ is solvable of length $\leq m+n$.

We will need some facts about wreath products. The first is a beautiful theorem of Kaloujnine and Krasner [4] (a proof can also be found in [10]), which says that $A \mathrm{Wr} B$ is universal for extensions of $A$ by $B$ :

Theorem. If $G$ is an extension of $A$ by $B$ then $G$ can be embedded in $A \operatorname{Wr} B$.

Actually we will need not only the fact that the embedding exists, but some information about "where it sends what", so we give the definition of the map. Denote the canonical homomorphism of $G$ onto $B$ by ${ }^{-}$; let $X$ be a transversal of $A$ (i.e. a set of coset representatives) and define a map $\langle>$ from $B$ to $G$ by sending $b \in B$ to the element of $X$ which represents it. Define $\theta: G \rightarrow A \operatorname{Wr} B$ by $g \theta=\bar{g} f_{g}$, where $f_{g} \in A^{B}$ is given by the function $f_{g}(b)=\left\langle b \bar{g}^{-1}\right\rangle g\langle b\rangle^{-1}$ for $b \in B$.

We will also need part of a result from a paper of Peter M. Neumann; we state the relevant part in the following form.

Theorem [6, Corollary 5.3, p. 352]. If $w \in A \mathbb{W}_{\mathrm{r}} B$ has the form $w=b f$ with $b \in B$ of infinite order and $f \in A^{B}$, and if $b \in A^{B}$, then there exists $g \in A^{B}$ such that $w^{-1} g^{-1} w g=h$.

Finally, for the proof of Theorem 2 we will need a result of $P$. Hall which says that finitely generated metabelian groups are residually finite. We defer discussion of this result to $\S 3$, where Theorem 2 is proved. 
2. Proof of Theorem 1. Since $T_{n}$ is a universal theory, $\operatorname{Mod}\left(T_{n \forall}\right)=\operatorname{Mod}\left(T_{n}\right)$; in particular every existentially complete element of $\Sigma_{n}=\operatorname{Mod}\left(T_{n \forall}\right)$ is a model of $T_{n}$, so our task in proving Theorem 1 is to show that the class of existentially complete models of $T_{n}$ is not elementary. As indicated above, we will do this by showing that the class is not closed under ultrapowers.

In their proof of the corresponding result for the theory $T$ of all groups, Eklof and Sabbagh used the result of G. Higman, B. H. Neumann, and H. Neumann which says that if $a, b$ are elements of a group $G$ and $a$ and $b$ have the same order, then there is an extension $H$ of $G$ in which $a$ and $b$ are conjugate to each other. However, if $G$ is solvable of length $\leq n$ then it is easy to see that it need not be possible to choose $H$ solvable of length $\leq n$, so this result will not serve us here. We replace it with the following proposition, forged out of the algebraic machinery indicated in $\$ 1$.

Proposition 1. If $G \in \Sigma_{n}, c \in G^{(n-1)}$, and $b \in G$ has infinite order modulo $G^{(n-1)}$, then there exists an extension $H$ of $G$ in $\Sigma_{n}$ and an element $g \in H$ such that $b^{-1} g^{-1} b g=c$.

Proof. Let $A=G^{(n-1)}, B=G / A$. Notice that $A W_{\mathrm{r}} B \in \Sigma_{n}$, and consider the Kaloujnine-Krasner embedding $\theta: G \rightarrow A \mathbb{W}_{\mathrm{r}} B$. $b \theta$ has the form $\bar{b} f$ with $\bar{b} \in B$ of infinite order; $c \theta \in A^{B}$. So by Peter Neumann's result,

$$
A W_{r} B \vDash \exists g\left((b \theta)^{-1} \circ g^{-1} \circ(b \theta) \circ g=c \theta\right) \text {. }
$$

An obvious isomorphism yields an extension $H$ of $G$ in $\Sigma_{n}$ such that

$$
H \vDash \exists g\left(b^{-1} g^{-1} b g=c\right) \text {. }
$$

Remark. In fact it is clear that $g$ can be chosen to be a commutator.

The next two propositions contain the algebraic information we will need about existentially complete (e.c.) elements of $\Sigma_{n}$.

Proposition 2. Let $n \geq 2$ and let $G$ be e.c. in $\Sigma_{n}$. Then for any prime q, $G$ contains an element of order $q$ which is not in $G^{(n-1)}$.

Proof. Let $A$ be any nontrivial abelian group and let $B$ be an abelian group containing an element $b$ of infinite order and an element $c$ of order $q$. In $A \mathbb{W r}_{\mathrm{r}} B$ pick an element $(1, f)$ of $A^{B}$ such that $(1, f)(c, 1) \neq(c, 1)(1, f)$, i.e. such that $f^{c} \neq f$; such an $f$ clearly exists because if $a \in B$ then $a c^{-1} \neq a$ (since $c \neq 1$ ) so since $A$ is nontrivial there exists $f \in A^{B}$ such that $f\left(a c^{-1}\right) \neq f(a)$, i.e. $f^{c}(a)$ $\neq f(a)$. By the presence of $b$ and Neumann's result, $(1, f)$ is a commutator in $A \mathbb{W}_{\mathrm{r}} B$. Thus $A \mathbb{W}_{\mathrm{r}} B$ contains an element $c$ of order $q$ which does not commute with some commutator $f$. 
Now again by Neumann's result, every element of $(A \mathrm{Wr} B)^{B}$ is a commutator in $\left(A W_{\mathrm{r}} B\right) W_{\mathrm{r}} B$. If we identify $A W_{\mathrm{r}} B$ with the set of constant functions in $\left(A \mathbb{W}_{\mathrm{r}} B\right)^{B} \subseteq\left(A \mathbb{W}_{\mathrm{r}} B\right) \mathbb{W}_{\mathrm{r}} B$, we see that $f$, which is already a commutator in $A \mathbb{W}_{\mathrm{r}} B$, becomes a second commutator in $\left(A \mathbb{W}_{\mathrm{r}} B\right) \mathbb{W}_{\mathrm{r}} B$, and $c$ is an element of order $q$ which does not commute with $f$. Proceed ing inductively, we see that if we let $H=\left(\ldots\left(\left(A \mathbb{W}_{\mathrm{r}} B\right) \mathbb{W}_{\mathrm{r}} B\right) \mathbb{W}_{\mathrm{r}} B \ldots\right) \mathbb{W}_{\mathrm{r}} B$, where $B$ is used $(n-1)$ times, then $H$ contains an element of order $q$ which fails to commute with some $(n-1)$ st commutator. It is clear that the existence of such an element can be expressed by a first-order existential sentence $\phi$.

Now since $H \in \Sigma_{n}, G \oplus H \in \Sigma_{n}$, and $G \oplus H \vDash \phi$. Since $G$ is e.c., this implies that $G \vDash \phi$. Thus $G$ contains an element of order $q$ which fails to commute with some $(n-1)$ st commutator; such an element is clearly not in $G^{(n-1)}$, since $G \in \Sigma_{n}$.

Proposition 3. Let $n \geq 2$ and let $G$ be e.c. in $\Sigma_{n}$. If $b \in G$, then for any prime $q$ there exists in $G$ an $(n-1)$ st commutator of order $q$ which commutes with b.

Proof. Let $A$ be an abelian group containing an element of order $q$, and let $B$ be an abelian group containing an element of infinite order. As in the proof of Proposition 2, $H=\left(\ldots\left(\left(A \mathbb{W}_{\mathbf{r}} B\right) \mathbb{W} \mathbf{r} B\right) \ldots\right) \mathbb{W}_{\mathbf{r}} B$ (where $B$ is used $(n-1)$ times) contains an $(n-1)$ st commutator or order $q$. Thus if we embed $G$ in $G \oplus H$, we see that $G \oplus H$ contains an $(n-1)$ st commutator of order $q$ which commutes with $b$. The existence of such an element can be expressed by an existential sentence $\psi$ involving a constant for $b$, and since $G$ is e.c. this sentence must hold in $G$.

We can now complete the proof of Theorem 1 .

Let $G \in \Sigma_{n}$ be e.c., and let $D$ be a nonprincipal ultrafilter on the set $I$ of positive integers. Then $G^{*}=G^{I} / D \in \Sigma_{n}$. Let $\left\{p_{i}\right\}_{i=1}^{\infty}$ be an enumeration in order of magnitude of the prime numbers. For $i \in I$ let $b_{i}$ be an element of $G-G^{(n-1)}$ of order $p_{2 i-1}$ (by Proposition 2) and let $c_{i}$ be an element of $G^{(n-1)}$ of order $p_{2 i}$ which commutes with $b_{i}$ (by Proposition 3). Let $b, c$ be the elements of $G^{*}$ represented by the sequences $\left\{b_{i}\right\}$ and $\left\{c_{i}\right\}$, respectively. Obviously, $c \in G^{*(n-1)}$. We claim that $b$ has infinite order modulo $G^{*(n-1)}$. For suppose $b^{k} \in G^{*(n-1)}$ for some integer $k$. Then there exist $(n-1)$ st commutators $x_{1}, \cdots, x_{r}$ in $G^{*}$ and a word $W\left(x_{1}, \ldots, x_{r}\right)$ such that $b^{k}=W\left(x_{1}, \ldots, x_{r}\right)$. (By a word $W\left(x_{1}, \ldots, x_{r}\right)$ we mean either 1 or an expression $d_{1} \epsilon_{1} \ldots d_{s} \epsilon_{s}$ where each $d_{i}$ is some $x_{j}$ and $\epsilon_{i}$ is an integer.) If $x_{j}$ is represented by $\left\{x_{j i}\right\}$ for $1 \leq j \leq r$ then for every $i$ in some set $F \in D, b_{i}^{k}=W\left(x_{1 i}, \ldots, x_{r i}\right)$ and each of $x_{1 i}, \ldots, x_{r i}$ is an $(n-1)$ st commutor. In particular $b_{i}^{k} \in G^{(n-1)}$ for every $i \in F$. But for any $i \in I, b_{i}^{k} \in G^{(n-1)}$ 
implies $p_{2 i-1} \mid k$, since $b_{i} \notin G^{(n-1)}$ has prime order $p_{2 i-1}$, so that its order modulo $G^{(n-1)}$ is $p_{2 i-1}$. Thus $F$ must be finite, contradicting the fact that $D$ is nonprincipal.

Now by Proposition 1 there exists an extension $H$ of $G^{*}$ in $\Sigma_{n}$ such that $H \vDash \exists g\left(b^{-1} g^{-1} b g=c\right)$. If $G^{*}$ is e.c., then $G^{*} \vDash \exists g\left(b^{-1} g^{-1} b g=c\right)$. If $\left\{g_{i}\right\}$ represents some $g \in G^{*}$ such that $b^{-1} g^{-1} b g=c$ then certainly for some $i$ we have

$$
\begin{gathered}
b_{i}^{-1} g_{i}^{-1} b_{i} g_{i}=c_{i}, \quad g_{i}^{-1} b_{i} g_{i}=b_{i} c_{i}, \\
g_{i}^{-1} b_{i}^{p 2 i-1} g_{i}=\left(b_{i} c_{i}\right)^{p} 2 i-1
\end{gathered}
$$

But $c_{i}$ was chosen to commute with $b_{i}$, and $b_{i}$ has order $p_{2 i-1}$, so we get

$$
1=b_{i}^{p_{2 i-1}} c_{i}^{p_{2 i-1}}, \text { i.e. } 1=c_{i}^{p_{2 i-1}}
$$

contradicting the fact that $c_{i}$ has order $p_{2 i}$. Thus $G^{*}$ is not e.c.; this completes the proof of Theorem 1.

3. Proof of Theorem 2. In this section we strengthen Theorem 1 for the case $n=2$, i.e. for the theory $T_{2}$ of metabelian groups.

We begin by recalling that a group $G$ is said to be residually finite if for any $g \neq 1$ in $G$, there exists a normal subgroup $H$ of finite index in $G$ such that $g \notin H$. It is easy to see, in fact, that if $G$ is residually finite and $g_{1}, \cdots, g_{s} \in G, g_{i} \neq 1$ for $1 \leq i \leq s$, then there is a normal subgroup $H$ of finite index such that $g_{i} \notin H$ for $1 \leq i \leq s$.

We will use the following special case of a result of Philip Hall [3].

Theorem. Every finitely generated metabelian group is residually finite.

We should point out that the corresponding result for groups solvable of length $\leq 3$, i.e. for $T_{3}$, is false (see [3]). This is why we have been able to strengthen Theorem 1 only for the case $n=2$. We would also like to express our thanks to Professor C. F. Miller for informing us of the existence of Hall's result at a time when it was just what we wanted to know.

To indicate the relevance of Hall's result to our aims we recall the notion of a finite forcing condition relative to $T_{2}$. A condition $p\left(c_{1}, \cdots, c_{n}\right)$ (for $c_{i}$ in some collection $C$ of new constant symbols) is a finite set of atomic and negated atomic sentences which is consistent with $T_{2}$, i.e. which is such that there exist elements $a_{1}, \cdots, a_{n}$ in some metabelian group $G$ such that $G$ satisfies the conjunction of the statements in $p$ when $c_{i}$ is interpreted as $a_{i}$. We can write 


$$
\begin{aligned}
& p\left(c_{1}, \ldots, c_{n}\right)=\left\{w_{1,1}\left(c_{1}, \ldots, c_{n}\right)=w_{1,2}\left(c_{1}, \ldots, c_{n}\right), \ldots,\right. \\
& \left.\quad W_{r, 1}\left(c_{1}, \ldots, c_{n}\right)=W_{r, 2}\left(c_{1}, \ldots, c_{n}\right)\right\} U, \\
& U\left\{W_{r+1,1}\left(c_{1}, \ldots, c_{n}\right)\right. \\
& \left.\neq W_{r+1,2}\left(c_{1}, \ldots, c_{n}\right), \ldots, w_{r+s, 1}\left(c_{1}, \ldots, c_{n}\right) \neq W_{r+s, 2}\left(c_{1}, \ldots, c_{n}\right)\right\}
\end{aligned}
$$

for some terms $W_{i j}$ in $c_{1}, \cdots, c_{n}$.

What Hall's the orem gives us is

Proposition 4. Let $p\left(c_{1}, \cdots, c_{n}\right)$ be a condition relative to $T_{2}$. Then there exists a positive integer $m$ such that $p \cup\left\{c_{1}^{m}=1, \ldots, c_{n}^{m}=1\right\}$ is a condition.

Proof. Let $a_{1}, \ldots, a_{n}$ be elements of some metabelian group $G$ such that $G$ satisfies all the elements of $p$ at $a_{1}, \ldots, a_{n}$. Let $G_{0}$ be the subgroup of $G$ generated by $a_{1}, \cdots, a_{n}$. In the above notation, $W_{r+j, 1}\left(a_{1}, \ldots, a_{n}\right) W_{r+j, 2}\left(a_{1}, \cdots, a_{n}\right)^{-1}$ $\neq 1$ in $G_{0}$ for $1 \leq j \leq s$. By Hall's result we can let $H$ be a normal subgroup of finite index in $G_{0}$ which excludes these $s$ elements. $G_{0} / H$ is finite, say of order m. Clearly if $a_{1}^{\prime}$ is the image of $a_{i}$ under the canonical homomorphism for each $i$ then $G_{0} / H$ satisfies all the elements of $p$ at $a_{1}^{\prime}, \ldots, a_{n}^{\prime}$, and each $a_{i}^{\prime}$ satisfies $\left(a_{i}^{\prime}\right)^{m}=1$.

Now let $\psi\left(x_{1}\right)$ be the formula $\forall x_{2} \forall x_{3} \exists x_{4} \exists x_{5}\left(\left[x_{2}, x_{3}\right]=\left[x_{1},\left[x_{4}, x_{5}\right]\right]\right)$, and let $\phi$ be $\exists x_{1} \psi\left(x_{1}\right)$.

Proposition 5. Let $G$ be any finitely generic metabelian group. Then $G \vDash \neg \phi$.

Proof. Suppose $G \vDash \phi$. Then there is a condition $p$ and a $c_{1} \in C$ such that

$$
p \Vdash \forall x_{2} \forall x_{3} \exists x_{4} \exists x_{5}\left(\left[x_{2}, x_{3}\right]=\left[c_{1},\left[x_{4}, x_{5}\right]\right]\right) \text {, }
$$

and we can assume that $c_{1}$ occurs in $p$. In particular, for any $p_{1} \supset p$ and any $c_{2}$, $c_{3}$ in $C$ there are $c_{4}$ and $c_{5}$ in $C$ and an extension $p_{2}$ of $p_{1}$ such that $p_{2} \mathbb{H}\left[c_{2}, c_{3}\right]=\left[c_{1},\left[c_{4}, c_{5}\right]\right]$. By Proposition 4 there is an integer $m$ such that $p_{0}=p \cup\left\{c_{1}^{m}=1\right\}$ is a condition. If we choose $c_{2}$ and $c_{3}$ not mentioned in $p$ then we see that $p_{1}=p_{0} \cup\left\{\left[c_{2}, c_{3}\right] c_{1}=c_{1}\left[c_{2}, c_{3}\right],\left[c_{2}, c_{3}\right]^{m} \neq 1\right\}$ is a condition. (Let $p_{0}$ be satisfied in $G_{0}$, let $G_{1}$ contain a commutator of order $q$ for some prime $q>m$, and consider $G_{0} \oplus G_{1}$, as in the proof of Proposition 3.) By the above there exist an extension $p_{2}$ of $p_{1}$ and $c_{4}, c_{5}$ such that $p_{2} \Vdash\left[c_{2}, c_{3}\right]=$ $\left[c_{1},\left[c_{4}, c_{5}\right]\right]$, i.e. $\left[c_{2}, c_{3}\right]=\left[c_{1},\left[c_{4}, c_{5}\right]\right] \in p_{2}$. Thus

$$
\left\{\left[c_{2}, c_{3}\right] c_{1}=c_{1}\left[c_{2}, c_{3}\right], c_{1}^{m}=1,\left[c_{2}, c_{3}\right]=\left[c_{1},\left[c_{4}, c_{5}\right],\left[c_{2}, c_{3}\right]^{m} \neq 1\right\}\right.
$$


is consistent with $T_{2}$, since it is contained in the condition $p_{2}$. But this is a contradiction, since the first three formulas in the set imply $\left[c_{2}, c_{3}\right]^{m}=1$, by the calculation that was used at the end of the proof of Theorem 1.

Remark. For the purposes of Propositions 5 and 6 we could simplify $\psi$ by deleting $x_{5}$ and replacing $\left[x_{4}, x_{5}\right]$ by just $x_{4}$. However in Proposition 7 we will want $\psi$ as it stands.

Proposition 6. Let $G$ be any infinitely generic metabelian group. Then $G \vDash \phi$.

Proof. Since $T_{2}$ has the joint embedding property, all infinitely generic metabelian groups are elementarily equivalent to each other (see [8]), so it suffices to find one infinitely generic $G$ such that $G \vDash \phi$.

Let $A$ be the cyclic group of order 2 and let $B$ be an infinite cycle on $b$. Let $G$ be any metabelian group extending $A$ Wr $B$. Then $b$ has infinite order modulo $G^{(1)}$. For suppose $b^{k} \in G^{(1)}$; then since $G$ is metabelian, $b^{k}$ commutes with every commutator in $G$. It is easy to see that $A \mathrm{Wr}_{\mathrm{r}} B$ is not abelian (cf. the proof of Proposition 2), so there is a nontrivial commutator $c$ in $A \operatorname{Wr} B$. Since $b^{k}$ has infinite order, Neumann's result implies that there exists a commutator $d$ in $A \operatorname{Wr}_{\mathrm{r}} B$ such that $c=\left(b^{k}\right)^{-1} d^{-1} b^{k} d$. Thus $d$ is a commutator with which $b^{k}$ does not commute and we have a contradiction.

We conclude, by the remark following Proposition 1 , that if $x$ is any commutator in $G$ then there exist a metabelian extension $H$ of $G$ and elements $y, z \in H$ such that $x=[b,[y, z]]$. Now assume that $G$ was chosen to be e.c.; then there are such elements $y, z$ in $G$. Since $x$ was an arbitrary commutator in $G$, we conclude that $G \vDash \psi(b)$, so $G \vDash \phi$.

In particular, we can choose $G$ to be infinitely generic; then $G \vDash \phi$, finishing the proof.

Propositions 5 and 6 yield the first statement of Theorem 2; the rest of the theorem follows immediately, as does the conclusion that the finite and infinite forcing companions $T_{2}^{f}$ and $T_{2}^{F}$ of $T_{2}$ are different.

Remark. It is a general fact that the e.c. structures coincide with the finitely generic ones iff every structure in $\Sigma$ has a finitely generic extension (see [1]). In the present case we have been able to give a fairly simple element of $\Sigma_{2}$, namely the wreath product of $\mathrm{Z}_{2}$ and an infinite cycle, which has no finitely generic extension. For as was shown in the proof of Proposition 6, any e.c. extension of this group satisfies $\phi$; but Proposition 5 tells us that every finitely generic metabelian group satisfies $7 \phi$.

\section{Further results on e.c. metabelian groups.}

Proposition 7. Let $G$ be an e.c. metabelian group, and $b \in G$. Then $G \vDash \psi(b)$ iff $b$ has infinite order modulo $G^{(1)}$. 
Proof. If $b$ has infinite order modulo $G^{(1)}$ then we showed during the proof of Proposition 6 that $G \vDash \psi(b)$. Conversely, if $b$ has order $n$ modulo $G^{(1)}$, then since $G$ is e.c. we see as before that there is in $G$ a commutator $c$ of prime order $q>n$ which commutes with $b$. Thus if $G \vDash \psi(b)$ there is a commutator $d \in G$ such that $b c=d^{-1} b d$, whence $b^{n} c^{n}=d^{-1} b^{n} d$, so since $b^{n}$ is in the commutator subgroup and $G$ is metabelian, $b^{n} c^{n} .=b^{n} d^{-1} d$, i.e. $c^{n}=1$, a contradiction.

Remark. Thus the property of being of infinite order modulo the commutator subgroup, which by the ultrapower argument in the proof of Theorem 1 is definitely not first-order relative to the class of all metabelian groups, is first-order relative to the class of e.c. ones.

Proposition 8. If $G$ is a finitely generic metabelian group then $G / G^{(1)}$ is periodic. If $G$ is an infinitely generic metabelian group then $G / G^{(1)}$ is not periodic.

Proof. By Propositions 5, 6, and 7.

In particular, no infinitely generic metabelian group is itself periodic. However, we have

Proposition 9. There exists a periodic finitely generic metabelian group.

Proof. Let $L(C)$ be the language obtained from $L$ by adding countably many new constants. Let $\left\{\phi_{i}\right\}_{i=1}^{\infty}$ be a listing of all the sentences in $L(C)$. Define a complete sequence $\left\{p_{i}\right\}$ of conditions as follows. Let $p_{1}$ be a condition which forces either $\phi_{1}$ or $7 \phi_{1}$. Let $p_{2}$ be obtained from $p_{1}$ by using Proposition 4, i.e. by adding statements which say that for some $m$ each constant used in $p_{1}$ denotes an element whose $m$ th power is 1 . If $i>2$ and $i$ is odd let $p_{i}$ be an extension of $p_{i-1}$ which forces either $\phi_{1 / 2(i+1)}$ or $7 \phi_{1 / 2(i+1)}$; if $i>2$ and $i$ is even let $p_{i}$ be obtained from $p_{i-1}$ by using Proposition 4 , as above. Let $G$ be the finitely generic metabelian group determined by the sequence $\left\{p_{i}\right\}$. Clearly $G$ is periodic.

Remarks. (1) The corresponding result is true for $T_{1}$ and false for $T$.

(2) Propositions 7 and 9 provide an alternate way of viewing the proof of Proposition 5, since all finitely generic metabelian groups are elementarily equivalent to each other.

(3) Propositions 2 and 7 imply that the direct product of infinitely many e.c. metabelian groups is never e.c.

(4) Obviously Propositions 4 and 9 have a general significance. For example, it is known that every finitely generated nilpotent group is residually finite. So we conclude that if $K_{n}$ denotes the theory of groups nilpotent of class $\leq n$, for any fixed $n$, then there exist periodic finitely generic models of $K_{n}$.

It is interesting to ask when a wreath product is itself existentially complete. 
For definiteness we ask when the wreath product of two abelian groups is an e.c. metabelian group. The next proposition will provide the answer.

Proposition 10. The center of an e.c. metabelian group is trivial.

Proof. Let $G \in \Sigma_{2}$ be e.c.; let $A=G^{(1)}, B=G / G^{(1)}$. Assume $g \neq 1$ in $G$. We claim that we can find an element in some metabelian extension of $G$ which does not commute with $g$; since $G$ is e.c. this will finish the proof.

Under the Kaloujnine-Krasner embedding of $G$ into $A W_{r} B, g$ becomes an element $(b, f)$ with $b \in B, f \in A^{B}$. We distinguish two cases.

Case (1): $b \neq 1$. Observe that $A$ is nontrivial since an e.c. metabelian group is clearly not abelian. As in Proposition 2, we let $a \in B$ and observe that $a b^{-1} \neq a$, so since $A$ is nontrivial there is $h \in A^{B}$ such that $b^{b} \neq b$, which implies that $(b, f)$ does not commute with $(1, b)$.

Case (2): $b=1$. Since $g \neq 1, b=1$ implies that there is some $a \in B$ such that $f(a) \neq 1$. Let $C$ be a proper abelian extension of $B$; then we can embed $A W_{\mathrm{r}} B$ into $A W_{\mathrm{r}} C$ by sending $(d, h)$ to $(d, k)$, where $k \in A^{C}$ has the value $h(x)$ for $x \in B$ and the value 1 for $x \in C-B$. Thus $(b, f)=(1, f)$ is mapped to $(1, t)$ where $t(x)=f(x)$ for $x \in B$ and $t(x)=1$ for $x \in C-B$. Let $y \in C-B$. Then $a y^{-1} \in C-B$ so $t\left(a y^{-1}\right)=1$, but $t(a)=f(a) \neq 1$, so $t^{y} \neq t$, which implies that $(y, 1) \in A \mathbb{W}_{\mathbf{r}} C$ does not commute with $(1, t)$.

Corollary, Let $A$ and $B$ be abelian. Then $A \mathbb{W}_{\mathrm{r}} B$ is never e.c. in $\Sigma_{2}$.

Proof. If $A$ is trivial $A \mathrm{~W}_{\mathrm{r}} B$ is abelian and so not e.c. in $\Sigma_{2}$. If $A$ is not trivial let $a \neq 1$ in $A$; then the element $f \in A^{B}$ such that $f(b)=a$ for all $b \in B$ is not the identity element of $A W_{r} B$, but it is easy to check that it is in the center. Thus $A \operatorname{Wr} B$ is not e.c., by Proposition 10.

Next, we prove

Proposition 11. If $G$ is an e.c. metabelian group, then both $G^{(1)}$ and $G / G^{(1)}$ are e.c. in $\Sigma_{1}$.

Proof. Probably the easiest approach is to use Eklof and Sabbagh's characterization of the class of e.c. abelian groups [2]. Eklof and Sabbagh proved that an abelian group is e.c. as an element of $\Sigma_{1}$ iff

(I) the group is divisible; and

(II) for every prime $p$ there are infinitely many elements of order $p$.

First consider $G^{(1)}$. Embed $G$ into $G^{(1)} W_{r} G / G^{(1)}$ by the Kaloujnine-Krasner map; let $E$ be an e.c. abelian group extending $G^{(1)}$, and embed $G^{(1)} \mathbb{W}_{\mathrm{r}} G / G^{(1)}$ into $E \mathrm{Wr} G / G^{(1)}$ in the obvious way; finally let $B$ be an abelian group extending $G / G^{(1)}$ and containing an element of infinite order, and embed $E W_{r} G / G^{(1)}$ into 
$E$ Wr $B$ as in Case (2) of the proof of Proposition 10. Under these maps an element $x$ of $G^{(1)}$ becomes an element of $E^{B}$; since $E$ and therefore $E^{B}$ is divisible by (I), for any positive integer $n$ there is in $E^{B}$ an element $y$ such that $y^{n}=x$. By Peter Neumann's result $y$ is a commutator in $E W_{\mathrm{r}} B$, so $E W_{\mathrm{r}} B \vDash$ $\exists u \exists v\left([u, v]^{n}=x\right)$; since $G$ is e.c. in $\Sigma_{2}, G \vDash \exists u \exists v\left([u, v]^{n}=x\right)$, i.e. $x$ is divisible by $n$ in $G^{(1)}$. Thus $G^{(1)}$ is divisible; the proof that $G^{(1)}$ satisfies (II) is similar.

Now consider $G / G^{(1)}$. Embed $G$ into $G^{(1)}$ Wr $G / G^{(1)}$. Let $E$ be an e.c. abelian group extending $G / G^{(1)}$ and containing an element of infinite order, and embed $G^{(1)} \operatorname{Wr} G / G^{(1)}$ into $G^{(1)} \operatorname{Wr} E$ as in Proposition 10. Notice that $\left(G^{(1)} \operatorname{Wr} E\right)^{(1)} \cap$ $G=G^{(1)}$. (The inclusion from right to left is obvious. For the other way, we observe that $\left(G^{(1)} W_{r} E\right)^{(1)} \subseteq G^{(1) E}$ since $\left(G^{(1)} W_{r} E\right) / G^{(1) E} \approx E$ is abelian. But by the definition of the Kaloujnine-Krasner embedding, the only elements of $G$ which end up in $G^{(1) E}$ under our sequence of maps are the elements of $G^{(1)}$.) Thus we have an obvious commutative diagram

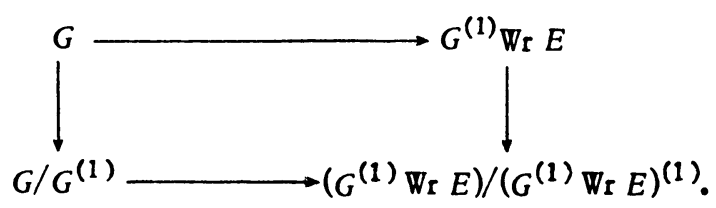

Now suppose $\bar{x} \in G / G^{(1)}$ is the image of $x \in G$ under the canonical homomorphism. It follows from the above parenthetical remarks and Neumann's result that $\left(G^{(1)} W_{\mathrm{r}} E\right)^{(1)}=G^{(1) E}$, so the group in the bottom right-hand corner of the diagram is isomorphic to $E$, and hence divisible. Thus this group satisfies $\exists y\left(y^{n}=\bar{x}\right)$, for any positive integer $n$. Thus

$$
G^{(1)} W_{\mathbf{r}} E \vDash \exists y \exists u \exists v\left(y^{n}=x[u, v]\right),
$$

so $G$ satisfies this sentence because it is e.c. in $\Sigma_{2}$. Thus $\bar{x}$ is divisible by $n$ in $G / G^{(1)}$, and we conclude that $G / G^{(1)}$ satisfies (I). That $G / G^{(1)}$ satisfies (II) can be seen by reconsidering the proof of Proposition 2.

Of course the converse of Proposition 11 is false, for otherwise the class of e.c. metabelian groups would be elementary in the wider sense (by Proposition 12 (ii)). More directly, if $A$ and $B$ are e.c. abelian groups and $B$ contains an element of infinite order then $H=A W_{\mathrm{r}} B$ is not e.c. by the corollary to Proposition 10 , but $H^{(1)}=A^{B}$ is e.c. by the Eklof-Sabbagh axioms and $H / H^{(1)} \approx B$ is also e.c.

Finally, we observe that whereas in any metabelian group $G$, the derived subgroup $G^{(1)}$ is an abelian normal subgroup $H$ such that $G / H$ is abelian, in an e.c. metabelian group the derived subgroup is characterized by this property. 
Proposition 12. In an e.c. metabelian group $G$,

(i) every element which commutes with every commutator is itself a commu• tator;

(ii) every element of $G^{(1)}$ is itself actually a commutator;

(iii) $G^{(1)}$ is the only abelian normal subgroup $H$ of $G$ such that $G / H$ is abelian.

Proof. (i) Embed $G$ into $G^{(1)} W_{r} B$ where $B$ is an abelian group extending $G / G^{(1)}$ and containing an element of infinite order. As in the proof of Proposition 10 , we see that any element of $G^{(1)} \operatorname{Wr} B$ which centralizes $G^{(1) B}$ must itself be in $G^{(1) B}$ and hence a commutator (by the choice of $B$ ). Hence

$$
G^{(1)} W_{\text {r } B} \vDash \forall x(\forall y \forall z(x[y, z]=[y, z] x) \rightarrow \exists u \exists v(x=[u, v])) \text {. }
$$

This sentence is equivalent to an $\forall \exists$ sentence, so since $G$ is e.c., the sentence holds in $G$.

(ii) Obvious from (i), since $G^{(i)}$ is abelian.

(iii) If $H$ is an abelian normal subgroup of $G$ such that $G / H$ is abelian, then clearly $H \supseteq G^{(1)}$, so since $H$ is abelian every element of $H$ commutes with every commutator in $G$. So $H \subseteq G^{(1)}$ by (i). Thus $H=G^{(1)}$.

In closing we remark that the principal results of this paper can be viewed as answering one form of the question of how far away one can go from the theory of abelian groups and still get a model companion. It would be interesting to have an answer to another form of this question, namely, what happens for the the ory of groups nilpotent of class $\leq c$ for fixed $c$ ?(2)

\section{REFERENCES}

1. J. Barwise and A. Robinson, Completing theories by forcing, Ann. Math. Logic 2 (1970), no. 2, 119-142. MR 42 \#7494.

2. P. Eklof and G. Sabbagh, Model-completions and modules, Ann. Math. Logic 2 (1970/71), no. 3, 251-295. MR 43 \#3105.

3. P. Hall, On the finiteness of certain soluble groups, Proc. London Math. Soc. (3) 9 (1959), 595-622. MR $22 \# 1618$.

4. L. Kaloujnine and $\mathrm{M}_{0} \mathrm{Krasner,} \mathrm{Le} \mathrm{produit} \mathrm{complète} \mathrm{des} \mathrm{groupes} \mathrm{de} \mathrm{permutations} \mathrm{et}$ le problème d'extension des groupes, C. R. Acad. Sci. Paris 227 (1948), 806-808. MR 10, 351.

5. A. Macintyre, On algebraically closed groups, Ann. of Math. (2) 96 (1972), 53-97.

6. P. M. Neumann, On the structure of standard wreath products of groups, Math. Z. 84 (1964), 343-373. MR 32 \#5719.

7. A. Robinson, Forcing in model theory, Symposia Mathematica, vol. V (INDAM,

$\left.{ }^{2}\right)$ The author has shown that for fixed $c \geq 2$ the theory of groups nilpotent of class $\leq c$ has no model companion. 
Rome, 1969/70), Academic Press, London, 1971, pp. 69-82. MR 43 \#4651.

8. - Infinite forcing in model theory, Proc. Second Scandinavian Logic Sympos., North-Holland, Amsterdam, 1971.

9. - Introduction to model theory and to the metamathematics of algebra, North-Holland, Amsterdam, 1965.

10. E. Schenkman, Group theory, Van Nostrand, Princeton, N. J., 1965. MR 33 \#5702.

DEPARTMENT OF MATHEMATICS, YALE UNIVERSITY, NEW HAVEN, CONNECTICUT 06520 